\title{
The Functional Synthesis of Linear Plots ${ }^{1}$
}

\author{
J. P. Vinti and R. F. Dressler ${ }^{2}$
}

(January 26, 1960)

\begin{abstract}
In practical engineering or experimental work one often encounters a function $F$ of many variables, $F$ ( $x$ 's, $y$ 's, $z$ 's), represented only by the families of curves obtained by plotting $F$ against each of the $x$ 's on Cartesian graph paper, against each of the $y$ 's on semilog paper, and against each of the $z$ 's on double-log paper. It frequently happens that these curves are all approximately straight lines over a limited range of interest. On the assumption that they are all true straight lines, the present note shows how to synthesize all the graphical representations, for any number of parameters, into the most general formula possible, expressing $F$ as the product of a multilinear function of the $x$ 's and the exponential of a constant-free multilinear function of the $y$ 's and of the log $z$ 's, the coefficients in both multilinear functions being independent of the $x$ 's, $y$ 's, and $z$ 's.
\end{abstract}

\section{Introduction}

In an investigation by one of the authors on assemblages of elastic shells, the various results for certain components of stress and displacement exhibited approximately linear behavior over the limited ranges of interest of the relevant parameters, when plotted on Cartesian graph paper, semi-log paper, or double-log paper. These results, derived from various numerical calculations and corroboratory experiments, are functions of many parameters, such as the various geometrical ratios defining shell shapes, the shell thickness, Poisson's ratio, the number of coupled shells, and other significant quantities. Rather than to retain these extensive results in the form of cumbersome families of graphs, it was desirable and useful to combine them in such a way as to obtain a single explicit formula for each dependent quantity, in terms of the above-mentioned independent parameters.

The problem of combining such results is, of course, not peculiar to investigations in elasticity but often arises in experimental or engineering work of any nature. One can very easily construct simple functions which will exhibit some of these "linear" properties, but the most general answer to the inverse problem is less obvious, especially when the number of parameters is large. Then an unsystematic attempt to effect such a synthesis may prove infeasible or incomplete, and furnish no assurance that one has indeed constructed the most general function with these properties. Our present note, therefore, formulates the general problem for any number of variables in a precise manner and derives its most general solution. For the simple case of only three parameters, the solution is illustrated by a few examples.

\section{The General Problem}

In the following developments we shall assume that all the pertinent quantities have been grouped into independent dimensionless combinations, so as to take advantage of whatever information is provided by the Buckingham Pi theorem. ${ }^{3}$ Let us then consider a dimensionless function $F$ of the dimensionless independent variables $x_{1}, x_{2}, \ldots, x_{m} ; y_{1}, y_{2}, \ldots ., y_{p} ; z_{1}, z_{2}, \ldots . ., z_{q}$; and $u_{1}, u_{2}, \ldots, u_{r}$, such that a straight line results when we plot $F$ against any $x$ on ordinary Cartesian graph paper, against any $y$ on semi-log paper, or against any $z$ on double-log paper. Let the $u$ 's denote all other dimensionless variables on which $F$ may depend, but for which no such "linear" property exists. It is understood, of course, that in practice such linearity may hold only approximately, and only for a certain bounded range of the variable used as abscissa, and only for certain bounded ranges of the remaining variables, which appear as parameters. With no loss of generality, however, we relax these restrictions, assuming that each plot is a straight line for all values of abscissa and parameters.

1 This research was supported by the United States Air Force, through the Office of Scientific Research of the Research and Development Command.

2 On leave to Institute of Mathematical Sciences, New York University, New York, N.Y.

${ }^{3}$ P. W. Bridgman, Dimensional analysis, p. 36 (Yale Univ. Press, New Haven, Conn., 1931). 
We put

$$
\ln z_{1}=y_{p+1}, \quad \ln z_{2}=y_{p+2}, \quad \ldots, \quad \ln z_{q}=y_{n}, \quad(n=p+q)
$$

then $F\left(x_{1}, \ldots x_{m}, y_{1} \ldots y_{n}, u\right)$ is a function such that the plot of $F$ against any $x$ or of $\ln F$ against any $y$ is a straight line on Cartesian graph paper. Here $u$ denotes the set $u_{1}, u_{2}, \ldots$., $u_{r}$. Then

$$
\ln F=A_{k} y_{k}+B_{k} \quad(k=1,2, \ldots, n)
$$

where $A_{k}$ and $B_{k}$ are functions of $x_{1} \ldots x_{m}, y_{1} \ldots y_{k-1}, y_{k+1} \ldots y_{n}$, and of the $u$ 's. We then ask: if $\ln F$ is linear in each $y$ when the other $y$ 's are all held constant, what is the most general form for $\ln F$, as a function of the $y$ 's, that will represent such a property?

To answer this preliminary question, we first recall the definition of a multilinear function $G$, of several variables $t_{1}, t_{2}, \ldots, t_{s}$, as a function which is the sum of a constant and a linear combination of all the products of the $t$ 's taken one at a time, two at a time, . ., s at a time, without repetition. For example, if $s=3$,

$$
G\left(t_{1}, t_{2}, t_{3}\right)=a_{0}+a_{1} t_{1}+a_{2} t_{2}+a_{3} t_{3}+b_{1} t_{2} t_{3}+b_{2} t_{3} t_{1}+b_{3} t_{1} t_{2}+c t_{1} t_{2} t_{3} .
$$

Any such multilinear function satisfies the differential equations

$$
\partial^{2} G / \partial t_{k}^{2}=0 \quad(k=1,2, \ldots, s) .
$$

(When the constant term vanishes, we term $G$ a constant-free multilinear function.) It is then easily shown by induction that $G\left(t_{1}, t_{2}, \ldots, t_{s}\right)$ is the most general function of $t_{1}, t_{2}, \ldots, t_{s}$ which is linear in each $t$.

\subsection{The Synthesis for the $y^{\prime}$ s Alone}

On applying these considerations to (2), we find that

$$
\ln F=g\left(x_{1}, x_{2}, \ldots, x_{m}, u\right)+N^{(x, u)}\left(y_{1}, y_{2}, \ldots, y_{n}\right),
$$

where $N^{(x, u)}$ denotes a general constant-free multilinear function of the $y$ 's, with coefficients which are, a priori, functions of $x_{1}, \ldots, x_{m}, u$. On placing

$$
\exp g=f\left(x_{1}, x_{2}, \ldots, x_{m}, u\right) \text {, }
$$

we obtain

$$
F=f\left(x_{1}, x_{2}, \ldots, x_{m}, u\right) \exp N^{(x, u)},
$$

which gives the synthesis of the linearities of $\ln F$ versus the $y$ 's.

\subsection{The Complete Synthesis}

Since $F$ is linear in each $x$, it follows from the property of multilinear functions that

$$
F=P^{(v, u)}\left(x_{1}, x_{2}, \ldots, x_{m}\right) \text {, }
$$

where $P^{(y, u)}$ is a general multilinear function of the $x$ 's, the coefficients being functions of the $y$ 's and the $u$ 's. Then

where by (4)

$$
F=f\left(x_{1}, x_{2}, \ldots, x_{m}, u\right) \exp N^{(x, u)}=P^{(y, u)}\left(x_{1}, x_{2}, \ldots, x_{m}\right),
$$

$$
\begin{aligned}
& \partial^{2} N / \partial y_{k}^{2}=0 \quad(k=1,2, \ldots, n) \\
& \partial^{2} P / \partial x_{j}^{2}=0 \quad(j=1,2, \ldots, m) .
\end{aligned}
$$


We next show that $f$ is a multilinear function of the $x$ 's. To do so, put every $y$ equal to zero in (9). Since $N$ contains no constant term, it then vanishes, so that (9) becomes

$$
f\left(x_{1}, x_{2}, \ldots, x_{m}, u\right)=M^{(u)}\left(x_{1}, x_{2}, \ldots, x_{m}\right) .
$$

Here $M^{(u)}$ is simply the expression for $P^{(y, u)}$ with each coefficient evaluated at each $y=0$, so that it is a multilinear function of the $x$ 's with coefficients depending only upon the $u$ 's.

We now show that the coefficients in $N^{(x, u)}$ are independent of the $x$ 's, as follows. Insert (12) into (7), so that

$$
F=M^{(u)} \exp N^{(x, u)}
$$

and require that (13) satisfy (11). It follows that

$$
\frac{\partial^{2} M}{\partial x_{j}^{2}}+M \frac{\partial^{2} N}{\partial x_{j}^{2}}+2 \frac{\partial M}{\partial x_{j}} \frac{\partial N}{\partial x_{j}}+M\left(\frac{\partial N}{\partial x_{j}}\right)^{2}=0
$$

where we have omitted the superscripts for convenience. Since $M$ is multilinear in the $x$ 's, it follows from (4) that

so that

$$
\partial^{2} M / \partial x_{j}^{2}=0,
$$

$$
M \frac{\partial^{2} N}{\partial x_{j}^{2}}+2 \frac{\partial M}{\partial x_{j}} \frac{\partial N}{\partial x_{j}}+M\left(\frac{\partial N}{\partial x_{j}}\right)^{2}=0
$$

If we now differentiate (16) twice with respect to $y_{k}$, we find with use of (10) that

$$
2 M\left(\frac{\partial^{2} N}{\partial x_{j} \partial y_{k}}\right)^{2}=0
$$

whence

$$
\frac{\partial}{\partial y_{k}}\left(\frac{\partial N}{\partial x_{j}}\right)=0\left\{\begin{array}{l}
j=1,2, \ldots, m \\
k=1,2, \ldots, n .
\end{array}\right.
$$

Equation (18) means that $\partial N / \partial x_{j}$ can be a function only of the $x$ 's and the $u$ 's. But $N$ and $\partial N / \partial x_{j}$ are constant-free multilinear functions of the $y$ 's, with coefficients that are, a priori, functions of the $x$ 's and the $u$ 's. These results are compatible if and only if

$$
\partial N / \partial x_{j}=0 \quad(j=1,2, \ldots, m)
$$

so that each coefficient in the constant-free multilinear function $N$ must be independent of the $x$ 's.

We may thus rewrite (13) as

$$
F=M^{(u)}\left(x_{1}, x_{2}, \ldots, x_{m}\right) \exp \mathrm{N}^{(u)}\left(y_{1}, y_{2}, \ldots, y_{n}\right) .
$$

When we return to the original formulation of the problem in terms of the $x$ 's, $y$ 's, and z's, it follows that the most general functional form for $F$ is given by

$$
F=M^{(u)}\left(x_{1}, x_{2}, \ldots, x_{m}\right) \exp \mathrm{N}^{(u)}\left(y_{1}, y_{2}, \ldots, y_{p}, \ln z_{1}, \ln z_{2}, \ldots, \ln z_{q}\right),
$$

where $M^{(u)}$ is a general multilinear function of the $x$ 's and $N^{(u)}$ a general constant-free multilinear function of the $y$ 's and lnz's, the coefficients in both being functions only of the $u$ 's.

\section{Some Elementary Examples}

As short illustrations of the general result (21), we append a few cases where $F$ has linear plots against only three variables. For each we list the specific form that (21) assumes and the slopes and intercepts on the appropriate plots. By comparing the behavior of the slopes. 
and intercepts in the various experimentally given families of curves that define the function $F$ with these listed formulas, one can readily determine which coefficients vanish, if any, and thus obtain a specific formula for $F$ in any actual case. Here "In" denotes a natural logarithm and "log" a common logarithm. For an $x$ or a $y$ the intercept is taken at zero, while for a $z$ it is taken at $z=1$. For the logarithmic plots the slopes and intercepts are those of $\log F$. In the following formulas, it is understood that the constants may be functions of the $u$ 's.

(a) $\quad \mathbf{x}, \mathbf{y}_{1}, \mathbf{y}_{2}$

$$
F=\left(k_{1} x+k_{2}\right) \exp \left(a_{1} y_{1}+a_{2} y_{2}+b y_{1} y_{2}\right)
$$

Cartesian plot versus" $x$ :

$$
\begin{array}{ll}
\text { slope } & S=k_{1} \exp \left(a_{1} y_{1}+a_{2} y_{2}+b y_{1} y_{2}\right) \\
\text { intercept } & I=k_{2} \exp \left(a_{1} y_{1}+a_{2} y_{2}+b y_{1} y_{2}\right)
\end{array}
$$

Semi-log plot versus $y_{1}$ :

$$
\begin{array}{ll}
\text { slope } & S=0.4343\left(a_{1}+b y_{2}\right) \\
\text { intercept } & I=\log \left(k_{1} x+k_{2}\right)+0.4343 a_{2} y_{2}
\end{array}
$$

(b)

$$
\mathbf{x}, \mathbf{y}, \mathbf{z}
$$

$$
F=\left(k_{1} x+k_{2}\right) e^{a_{1} y} z^{a_{2}+b y}
$$

Cartesian plot versus $x$ :

$$
\begin{array}{ll}
\text { slope } & S=k_{1} e^{a_{1} y} z^{a_{2}+b y} \\
\text { intercept } & I=k_{2} e^{a_{1} y} z^{a_{2}+b y}
\end{array}
$$

Semi-log plot versus $y$ :

$$
\begin{array}{ll}
\text { slope } & S=0.4343 a_{1}+b \log z \\
\text { intercept } & I=\log \left(k_{1} x+k_{2}\right)+a_{2} \log z
\end{array}
$$

Double-log plot versus $z$ :

$$
\begin{array}{ll}
\text { slope } & S=a_{2}+b y \\
\text { intercept } & I=\log \left(k_{1} x+k_{2}\right)+0.4343 a_{1} y
\end{array}
$$

(c) $\quad \mathbf{y}_{1}, \mathbf{y}_{2}, \mathbf{z}$

$$
F=k e^{a_{1} y_{1}+a_{2} y_{2}+b_{3} y_{1} y_{2}} z^{a_{3}+b_{1} y_{1}+b_{2} y_{2}+c y_{1} y_{2}}
$$

Semi-log plot versus $y_{1}$ :

$$
\begin{array}{ll}
\text { slope } & S=0.4343\left(a_{1}+b_{3} y_{2}\right)+\left(b_{1}+c y_{2}\right) \log z \\
\text { intercept } & I=\log k+0.4343 a_{2} y_{2}+\left(a_{3}+b_{2} y_{2}\right) \log z
\end{array}
$$

Double-log plot versus $z$ :

slope

intercept

(d)

$$
\mathrm{y}, \mathrm{z}_{1}, \mathrm{z}_{2}
$$

$S=a_{3}+b_{1} y_{1}+b_{2} y_{2}+c y_{1} y_{2}$

$I=\log k+0.4343\left(a_{1} y_{1}+a_{2} y_{2}+b_{3} y_{1} y_{2}\right)$

$$
F=k e^{a_{1} y} z_{1}^{a_{2}+b_{1} y} z_{2}^{a_{3}+b_{2} y} H
$$


where

$$
H=z_{1}{ }^{\left(b_{3}+c y\right) l n z_{2}}=z_{2}{ }^{\left({ }_{3}+c y\right) l n z_{1}}
$$

Semi-log plot versus $y$ :

$$
\begin{array}{ll}
\text { slope } & S=0.4343 a_{1}+b_{1} \log z_{1}+b_{2} \log z_{2}+2.303 c \log z_{1} \log z_{2} \\
\text { intercept } & I=\log k+a_{2} \log z_{1}+a_{3} \log z_{2}+2.303 b_{3} \log z_{1} \log z_{2}
\end{array}
$$

Double-log plot versus $z_{1}$ :

$$
\begin{array}{ll}
\text { slope } & S=a_{2}+b_{1} y+2.303\left(b_{3}+c y\right) \log z_{2} \\
\text { intercept } & I=\log k+0.4343 a_{1} y+\left(a_{3}+b_{2} y\right) \log z_{2}
\end{array}
$$

Washington, D.C.

(Paper 64C2-31) 